\title{
Juventud andaluza y emancipación ¿Independencia residencial y/o autosuficiencia económica?
}

\author{
Marta Donat López \\ María Dolores Martín-Lagos López \\ Universidad de Granada. Departamento de Sociología \\ martalou89@correo.ugr.es; lmlagos@ugr.es
}

Recepción: 07-09-2018

Aceptación: 26-01-2019

Publicación: 26-07-2019

\section{Resumen}

La actualidad española se caracteriza por un retraso en el calendario en lo que respecta al logro de una emancipación completa, consecuencia de un alargamiento en el periodo formativo y de una inestabilidad y una precariedad considerables en el empleo, entre otras cuestiones. Concretamente, Andalucía es una de las comunidades autónomas con más problemas en este sentido - mayor nivel de desempleo y de precariedad laboral, entre otros aspectos-. El objetivo principal del presente artículo es el de conocer los factores que influyen en la probabilidad de que un joven se emancipe. Para ello se propone distinguir, dentro de la emancipación, cuáles son independientes residencialmente y/o económicamente $y$, de esta forma, se persigue averiguar si existen diferencias en los factores que influyen en que un joven sea independiente residencialmente y no económicamente, y viceversa. Se ha utilizado una base de datos de 2017, con una muestra de 1.981 jóvenes de 14 a 35 años. La regresión logística multinomial ha sido la técnica de análisis utilizada. Los resultados muestran que los jóvenes que se aproximan más a la finalización de la transición de la juventud tienen más probabilidades de haber formado un hogar propio y de ser autosuficientes económicamente. Sin embargo, aquellos que todavía están formándose o tienen intención de retomar los estudios, están desempleados y no tienen descendencia ni intención de tenerla, se aproximan más a formas de independencia incompletas (residencial o económica) o bien nulas.

Palabras clave: jóvenes; Andalucía; España; emancipación; independencia económica; independencia residencial; regresión multinomial 


\title{
Abstract. Andalusian youth: Residential independence andlor economic self-sufficiency?
}

In recent years, the situation of youth in Spain has been characterized by delayed residential and economic independence as the result of longer training periods, as well as job instability and precariousness, among others. Specifically, Andalusia is one of the regions that most suffers from high levels of unemployment and job insecurity. The main aim of this article is to identify factors that influence the probability that a young person will become emancipated. To this end, we distinguish between young people who are residential and/or economically independent and determine which factors influence whether a young person is residential but not economically independent, and vice versa. The analysis is carried out using a 2017 database with a sample of 1,981 young people aged 14 to 35 years. The analysis is performed using multinomial logistic regression. The results show that people near the end of the transition from youth are more likely to have formed their own home and be financially self-sufficient. However, young people who are still in training or intend to return to education, are unemployed or have no offspring or intention of having one, are more likely to display incomplete forms of independence (residential or economic) or none at all.

Keywords: youth; Andalusia; Spain; emancipation; economic independence; residential independence; multinomial regression

\author{
Sumario \\ 1. Introducción 6. Resultados \\ 2. Marco teórico 7. Conclusiones \\ 3. Hipótesis, variables y metodología Agradecimientos \\ 4. Variable dependiente Referencias bibliográficas
}

5. Variables independientes

\section{Introducción}

En los últimos años ha descendido de forma considerable la tasa de emancipación entre los jóvenes españoles, sobre todo en el caso de los que se encuentran entre los 16 y los 29 años, no tanto en tramos superiores de edad (Consejo de la Juventud de España, 2016). Ciertos cambios demográficos de la sociedad española han influido en el retraso de la emancipación, como, por ejemplo, los siguientes: menos matrimonios y más tardíos, la convivencia en un mismo hogar de varias generaciones como nueva forma de hábitat, familias con dos sustentadores económicos y familias monoparentales. Sin embargo, el retraso del calendario en la natalidad es el rasgo principal de esta segunda transición demográfica. Según Lesthaeghe y Van de Kaa (1986), se trata de un proceso irreversible que tiene como consecuencia un alargamiento del periodo previo a la formación de una familia y de un hogar propio, el cual es cubierto por un mayor tiempo de formación y de consolidación de la vida profesional (Devolder y Tejada, 2007). 
Además de la prolongación de la etapa formativa, una serie de circunstancias vinculadas a la complejidad del mercado laboral y a la situación económica han derivado en ese retraso en el calendario de salida del hogar de sus progenitores. Esta situación resulta de especial interés en España, país caracterizado por unos fuertes lazos familiares y por un sistema de bienestar que proporciona escasas ayudas económicas dirigidas a los jóvenes. Según Esping-Andersen (1999), en España, ejemplo de régimen de bienestar orientado a la familia, las redes familiares de asistencia son fuertes y asumen la responsabilidad del apoyo financiero y social cuando es necesario, por lo que se puede hablar de un modelo familista en el sur de Europa. Es por ello que, en España, la cohabitación es la principal estrategia de apoyo a los jóvenes emancipados con problemas para mantener su independencia (Albertini, 2010).

A todo lo anteriormente expuesto hay que sumarle las condiciones laborales de los jóvenes españoles. La precariedad en el empleo da lugar a que la transición a la vida adulta se encuentre sometida a diferentes periodos en los que ellos circulan por el mercado de trabajo en busca de esa estabilidad.

Concretamente, Andalucía destaca por ser una de las comunidades autónomas donde los jóvenes más postergan la salida del hogar. A fecha de 2017 tiene la cuarta tasa de emancipación más baja en España (Consejo de la Juventud de España, 2017), debido a su frágil posicionamiento en el mercado de trabajo, y ocupa la tercera tasa de desempleo más alta del territorio español (Consejo de la Juventud de España, 2017). Además, destaca por ser una comunidad que está por debajo de la media española en lo que respecta al nivel formativo; concretamente, ocupa uno de los últimos lugares en referencia al porcentaje de población con estudios superiores, solo por detrás de Castilla-La Mancha y Extremadura (INE, 2018).

No obstante, no solo las necesidades económicas son los factores que promueven la postergación de la marcha del hogar de los progenitores por parte de los jóvenes, sino también la búsqueda de seguridad y de apoyo emocional. La comodidad y la calidad de vida son cualidades que se tienen muy en cuenta a la hora de retrasar la emancipación, así como también para regresar al hogar familiar (Goldscheider y Goldscheider, 1999).

Por tanto, muchos son los factores, tanto a nivel macro como a nivel micro, que influyen en la decisión emancipatoria. Entre estos últimos, diversas investigaciones - como se indica a posteriori de forma detallada - señalan una serie de variables. Así, aspectos como la edad, el sexo, estar estudiando o trabajando, tener pareja estable o hijos, así como también la clase social y la estructura del hogar, entre otros, están relacionados con el calendario emancipatorio.

En este contexto, el objetivo del presente artículo consiste en dar respuesta a la siguiente pregunta: «¿Cuáles son los factores que influyen o determinan en mayor medida el hecho de que un joven o una joven se emancipe?». Para responder a esta cuestión, en esta investigación se distingue, dentro de la emancipación, qué jóvenes son independientes residencialmente y/o económicamente $y$, de esta forma, se persigue averiguar si existen diferencias en los factores que 
influyen en el hecho de que un joven sea independiente residencialmente y no económicamente, o viceversa. Así, una cuestión esencial que aporta este estudio radica en que no solo se estudia la emancipación a partir de la independencia residencial de los jóvenes, sino también de la económica.

Otra cuestión reseñable es que se ha utilizado una nueva base de datos, todavía no explotada, elaborada para llevar a cabo el diseño del III Plan Integral de Juventud de Andalucía. Se cuenta con 1.981 jóvenes de entre 14 y 35 años pertenecientes a cada una de las provincias andaluzas.

Una vez expuestos los objetivos de esta investigación y de haber realizado una introducción al estado de la cuestión, se desarrolla un marco teórico en el que se contextualiza la emancipación en el contexto de España y de Andalucía, así como también las variables socioeconómicas y contextuales que influyen en los jóvenes en la decisión de emanciparse. Posteriormente, se enuncian las hipótesis, así como la metodología utilizada. Finalmente, se exponen los resultados más significativos en función de los objetivos y de las hipótesis del estudio, para posteriormente extraer conclusiones y recomendaciones para futuras líneas de investigación.

\section{Marco teórico}

\subsection{Factores individuales, familiares y contextuales que influyen en la decisión emancipatoria}

En primer lugar, respecto a las variables influyentes en la decisión de emanciparse relativas a características sociodemográficas de los jóvenes, vemos como algunos autores indican que la edad en la que se encuentren está relacionada con el hecho de salir del hogar de los progenitores (Billari y Liefbroer, 2007). Otros investigadores, como por ejemplo Mitchell et al. (2017), Colom y Molés (2016) o Bellart y Oller (2005), indican que el comportamiento de los jóvenes ante la salida del hogar familiar varía entre hombres y mujeres.

El periodo de escolarización o etapa formativa se señala como otra de las variables independientes que afectan al calendario de la emancipación, concretamente, encontrarse dentro de dicha fase posterga el abandono del hogar (Miret, 2006; Jones, 1995; Colom y Molés, 2016; Bellart y Oller, 2005; Ballesteros et al., 2012; South y Lei, 2015).

Respecto a cuestiones relacionadas con el empleo, Miret (2006) y Ghidoni (2002), entre otros, indican la importancia de la actividad como predictora de la emancipación; por ejemplo: estar desempleado, ocupado o cobrando una pensión. Concretamente, algunas investigaciones apuntan que los jóvenes abandonan el hogar familiar en mayor medida si disponen de trabajo o si no se encuentran en condiciones laborales precarias (Iacovou, 2010; Ghidoni, 2002; Colom y Molés, 2016'; Bellart y Oller, 2005; Moreno, 2012). Autores

1. Colom y Molés (2016) indican que en las mujeres no ocurre tanto, puesto que aun siendo inactivas se emancipan. Este hecho lo relacionan con el rol de ama de casa que siguen ejerciendo ellas. 
como Becker et al. (2010) hablan concretamente de la inseguridad laboral al respecto. También adquiere cierto énfasis la variable relativa a los ingresos. Autores como Jones (1995), Ghidoni (2002) y Ermisch (1999) señalan que la reducción de las oportunidades de una obtención de ingresos regulares para los jóvenes influye negativamente en la emancipación, y viceversa. Sin embargo, Jones (1995) indica que los jóvenes salen del hogar familiar a pesar de su falta de recursos económicos y aún con mayor riesgo de quedarse sin hogar.

Por otra parte, se recalca que la formación de una pareja estable favorece su emancipación (Huinink y Konietzka, 2000; South y Lei, 2015), así como también la formación de una familia propia. Convertirse en padre o en madre está estrechamente relacionado con el hecho de abandonar el hogar de los progenitores (Goldscheider et al., 2014; Holdsworth et al., 2002).

En segundo lugar, con respecto a las variables relativas al origen y, concretamente, a la familia de los jóvenes en cuestión, la clase social aparece como circunstancia que afecta a la emancipación (Parisi, 2008; Mykyta y Bureau, 2012; Moreno et al., 2012; Ballesteros et al., 2012). En particular, se incide en que una mayor cantidad de ingresos familiares desalientan las salidas del hogar de los progenitores (Ghidoni, 2002; Avery et al., 1992³; Manacorda y Moretti, 2006).

Resulta interesante la aportación de algunos investigadores como Calvo (2002) y Bernardi (2007) acerca de la importancia de las estrategias familiares de ascenso social como influyentes en la emancipación. Según este modelo, una condición para que los individuos se emancipen es que hayan alcanzado como mínimo la misma posición socioeconómica de sus familias de origen.

En relación con la ayuda monetaria proporcionada por los familiares a los jóvenes para llevar a cabo la emancipación, Jones (1995) indica que es decisiva con respecto al hecho de abandonar el hogar de los progenitores. Por su parte, South y Lei (2015) señalan que la ayuda económica familiar aumenta la probabilidad de emanciparse.

Estos últimos investigadores indican que un mal estado de salud de los progenitores no influye en la probabilidad de que los jóvenes se emancipen.

Mitchell et al. (2017) concede importancia al número de miembros del hogar como variable influyente en el hecho de que un joven se emancipe. Por otro lado, Blaauboer y Mulder (2010) indican que vivir en familias reconstituidas o en familias monoparentales aumenta el riesgo de abandonar el hogar de los progenitores, por lo que atribuyen un papel importante a la estructura doméstica al respecto. Estos mismos incorporan la dimensión del clima familiar como influyente; señalan que una atmósfera agradable en el domicilio disminuye la probabilidad de abandonarlo.

2. Ghidoni (2002) hace referencia al sur de Europa en todas las ocasiones en las que se menciona.

3. Avery et al. (1992) indican que una mayor cantidad de ingresos familiares disminuye o incrementa la probabilidad de que los jóvenes abandonen el domicilio familiar, dependiendo de la ruta de salida del hogar bajo consideración (matrimonio o independencia residencial prematrimonial). 
Bernardi (2007) advierte que en determinados lugares la raza o la etnia influyen en la marcha o en el retorno de los jóvenes respecto al domicilio familiar.

Finalmente, las circunstancias o las variables señaladas como predictoras de que se lleve a cabo el proceso de independencia por parte de los jóvenes relativas al contexto son, en primer lugar, la situación del mercado de vivienda (Patón Casas, 2007; Modena y Rondinelli, s. f.; Jones, 1995; Bellart y Oller, 2005; Ballesteros et al., 2012; Ermisch, 1999; Martins y Villanueva, 2009). Se subraya que, a mayor encarecimiento de la vivienda, menor es la probabilidad de abandonar el hogar. Moreno (2012) atribuye cierta importancia a la solidaridad intergeneracional y a las políticas de emancipación a la hora de tomar dicha decisión.

Las circunstancias económicas del contexto también son señaladas como determinantes respecto al calendario en el que se abandona el hogar familiar (Mykyta y Bureau, 2012; Moreno et al., 2012; Colom y Molés, 2016; Ballesteros et al., 2012). Por otra parte, las limitadas oportunidades que se dan en el actual mercado laboral desalientan la salida del hogar de los progenitores. Aspecto señalado por algunos autores como Ghidoni (2002), Ballesteros et al. (2012) y Moreno (2012).

Finalmente, según Jones (1995), el hecho de que el hogar familiar se encuentre en una zona rural o urbana no determina el calendario en la emancipación.

\subsection{La emancipación mediante una perspectiva comparada}

La literatura ha mostrado que existe una gran variación internacional respecto al momento de salir del hogar familiar, por lo que la misma se ha centrado principalmente en explicar por qué esto sucede así y ha encontrado grandes variaciones de unos países a otros. El patrón de salir de casa para establecer un hogar independiente es muy variable entre los estados europeos (Aasve et al., 2002).

Durante el periodo de expansión económica la emancipación fue un fenómeno creciente, tanto en el número de jóvenes que se lanzaban a ella como en la cada vez más temprana edad en la que se llevaba a cabo. El siguiente dato refleja este hecho: el porcentaje de españoles y españolas entre 18 y 34 años que abandonaron el hogar de sus progenitores pasó del 35,1\% en 2002 al 44,9\% en 2007 (Moreno y Rodríguez, 2012). Sin embargo, desde principios de 2008 la crisis económica empujó a proporciones crecientes de jóvenes emancipados a regresar al hogar de sus progenitores para convivir con ellos de nuevo.

Algunos informes muestran el progresivo retraso de la emancipación que se ha producido en general y la gran diferencia que se da entre distintos países de Europa, donde España, tras Grecia, destaca como el segundo lugar donde la emancipación es más tardía. Veamos algunas desemejanzas entre países: al concluir 2014, la media europea respecto a la edad de emancipación rondaba los 26,2 años; la de España, los 29,1, y la de Suiza, los 20,8 (un contraste de 
casi 10 años de edad). En el segundo trimestre de 2015 se sigue contemplando una disminución en el número de personas menores de 30 años que se han emancipado en España (Consejo de la Juventud de España, 2015).

Datos de la evolución al respecto indican un incremento de jóvenes emancipados hasta el inicio de la crisis económica, a partir de la cual la tendencia se invirtió hacia el descenso. Concretamente, en el cuarto trimestre de 2004 el número de personas de 18 a 34 años que se independizaron se incrementó en un 5,6\% respecto al año anterior; comparando con 2008, a finales de este año el aumento fue tan solo del 1,5\% (Gentile, 2010), por lo que se trata de un fenómeno muy susceptible a los ciclos económicos.

Respecto a la comunidad autónoma andaluza, los datos del OBJOVI muestran que la tasa de emancipación ha disminuido en los últimos años, aunque se observa una pequeña subida en 2016. Según grupos de edad, los jóvenes de 18 a 24 años y de 25 a 29 años muestran una tendencia claramente decreciente entre 2010 y 2012, mientras que en 2016 y 2017 ocurre lo contrario: se emancipan en mayor medida y la tendencia está en ascenso. Sin embargo, el intervalo de 30 a 34 años presenta una tasa de emancipación que no ha dejado de disminuir desde 2010 hasta la actualidad. Por tanto, este último tramo de edad parece ser el más perjudicado por el fenómeno del retraso emancipatorio.

Según los informes del Observatorio de Emancipación respecto a Andalucía en el segundo trimestre de 2016 referidos a jóvenes de entre 16 y 34 años, la tasa de emancipación se sitúa tan solo en el 18,1\%, un 3\% menos que un año atrás. Esto representa, junto al País Vasco, la cuarta tasa de emancipación más baja de España. Por tramos de edad, los datos manifiestan la heterogeneidad de este colectivo. Así, en los jóvenes de edades comprendidas entre 30 y 34 años esta tasa se sitúa en el 74,9\%; entre los jóvenes de 25 y 29 años pasa a ser el $44,2 \%$, y en los de entre 18 y 24 años, el $10 \%$.

Tabla 1. Posibles interpretaciones sobre el retraso de la emancipación

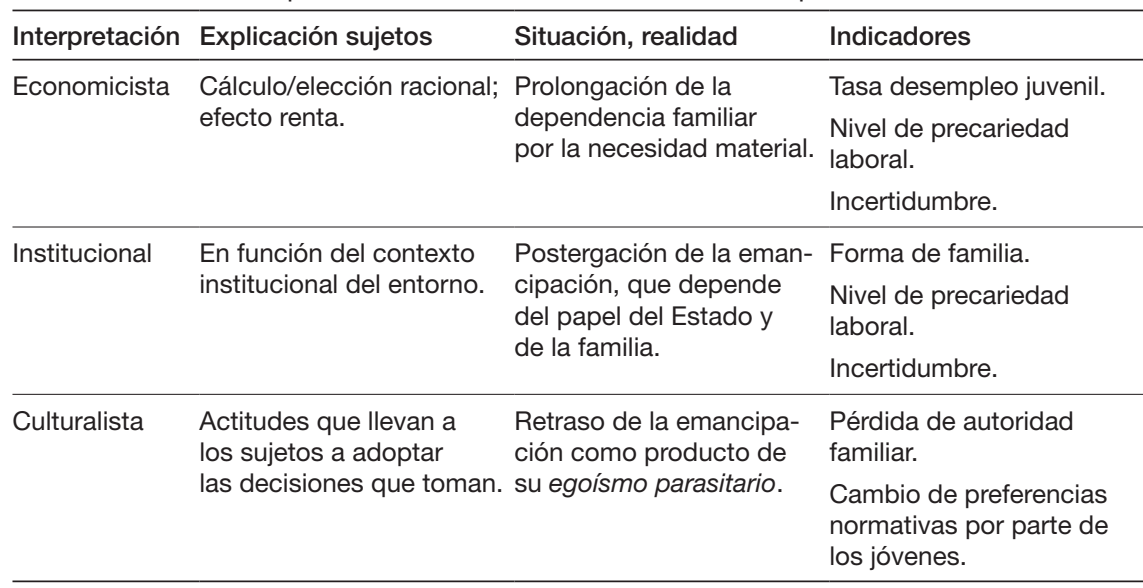

Fuente: elaboración propia a partir de Calvo (2002). 
En definitiva, queda constancia de que la emancipación se caracteriza por el retraso de la misma y por su postergación en el calendario, sobre todo en los países del sur de Europa y, concretamente, en Andalucía. No obstante, no solo resultan determinantes las teorías economicistas al respecto - como, por ejemplo, señalar a la crisis económica o a los ciclos económicos como factor único-, sino que existen varias explicaciones. Siguiendo a Calvo (2002), las agrupamos en función de la interpretación (tabla 1).

Teniendo en cuenta dichas interpretaciones, seguidamente se profundiza acerca de las causas o de los determinantes respecto al hecho de que un joven o una joven se emancipe o no. A continuación se muestra una revisión de la literatura de los últimos años que indica qué variable influye en la decisión emancipatoria, a partir de la cual se lleva a cabo el análisis de este estudio.

\section{Hipótesis, variables y metodología}

Las hipótesis de las que parte el análisis de dicho estudio devienen de la revisión bibliográfica realizada; concretamente, de las variables que influyen en la emancipación relativas a las condiciones sociodemográficas de los jóvenes en cuestión y de sus familias o progenitores. Cabe puntualizar que no se tienen en cuenta todos esos factores, a pesar de la importancia que suscita cada uno de ellos. En primer lugar, debido a la gran cantidad de variables y, en segundo lugar, a causa de los datos de los que disponemos a partir de la base de datos trabajada. Además, en Andalucía llama la atención sobre todo la alta cota de desempleo, así como el menor nivel de estudios que existe con respecto al resto de España y de Europa, por lo que se hace especial hincapié en estos factores.

H1. Ciertas variables sociodemográficas de los jóvenes están relacionadas con el hecho de emanciparse en Andalucía.

H1.1. El sexo y el nivel de estudios son variables que influyen en el hecho de tomar la decisión de abandonar el hogar de los progenitores en Andalucía.

H1.2. Dentro de la condición sociolaboral, estar ocupado favorece el abandono del hogar familiar por parte de los jóvenes andaluces, así como también el hecho de tener una pareja estable y tener hijos, o bien manifestar intención de tenerlos.

H1.3. Por el contrario, cuestiones como la intención de seguir estudiando por parte de los jóvenes andaluces que han finalizado su etapa educativa en algún momento, así como también encontrarse dentro de la categoría de estudiante o estar desempleado desalienta la emancipación.

H2. Las variables que influyen en la emancipación de los jóvenes andaluces son divergentes, al tener en cuenta, por un lado, la emancipación completa y, por otro, la independencia económica y no residencial, así como también la independencia residencial y no económica.

La encuesta ha sido efectuada por vía telefónica, mediante respuestas cerradas, con una duración media de 20 a 25 minutos. El muestreo utilizado es el polietápico estratificado. La muestra representativa asciende a 1.981 jóvenes de 14 a 
35 años, con reparto proporcional a la población de cada provincia (siguiendo criterios de dispersión geográfica), distribuidos por toda la comunidad autónoma andaluza y seleccionados siguiendo cuotas de edad, sexo y tamaño del municipio ${ }^{4,}$ 5. Para un nivel de confianza del 95,5\% (2 sigmas), en el caso más desfavorable $(p=q=0,50)$ y en el supuesto de muestreo aleatorio simple, el error es del $\pm 1,8 \%$ para el conjunto de la Comunidad Autónoma de Andalucía.

Para esta investigación se han seleccionado 1.710 sujetos de entre 18 y 35 años. Nos interesan aquellos de edad igual o superior a los 18 años, puesto que se está considerando la emancipación como tema central y, aunque puede que haya algún que otro caso de un joven que se haya emancipado con anterioridad, se trata de un fenómeno aislado. Se ha seleccionado ese amplio intervalo de edad tras una revisión exploratoria sobre cuándo se emancipan los jóvenes y se ha comprobado que esta se sitúa más en el último intervalo que en el de 18 a 24 años.

Para obtener los resultados se ha llevado a cabo un modelo de emancipación mediante una regresión logística multinomial. Este análisis permite conocer la probabilidad que tienen los jóvenes, en función de una serie de características sociodemográficas (variables independientes), de: 1) estar emancipado; 2) ser económicamente independiente, pero no residencialmente, y 3) ser residencialmente independiente, pero no económicamente (variable dependiente). A partir de dicho análisis se controlan cada una de las variables independientes, absteniéndolas de ser influenciadas por posibles alteraciones que puedan provocar las demás variables en ellas.

Sin embargo, este tipo de análisis no permite comparar las diferentes categorías de la variable dependiente: estar completamente emancipado o bien gozar únicamente de independencia económica o residencial. Para ello se ha optado por realizar la interpretación de modelos a través del análisis de los efectos marginales medios. De esta forma se obtiene información acerca de cómo las variables independientes aumentan o disminuyen la probabilidad de encontrarse dentro de cada una de estas categorías (Torrado, 2018). Dichos efectos marginales se presentan mediante una tabla que indica los resultados en porcentajes. Las interpretaciones presentan la probabilidad asociada a cada categoría de las variables explicativas de cada situación de independencia de los jóvenes.

\section{Variable dependiente}

Puesto que la emancipación como tal no aparece en la encuesta, se ha creado una variable a partir de la independencia residencial y económica que simule el hecho emancipatorio. En primer lugar, se mide la cuestión de independencia residencial preguntando: «¿Con quién vives?» ${ }^{6}$. Las categorías de respuesta

4. Los estratos del tamaño de municipio se distribuyen en el siguiente número de habitantes: hasta 100 residentes, de 101 a 500 , de 501 a 1.000 , de 1.001 a 2.000 , de 2.001 a 5.000 , de 5.001 a 10.000 , de 10.001 a 20.000 , de 20.001 a 50.000, de 50.001 a 100.000 y más de 100.000 .

5. Los municipios han sido seleccionados mediante rutas aleatorias.

6. Con ello nos referimos al núcleo de relaciones familiares, con independencia de que haya o no otras personas en el hogar. 
Tabla 2. Construcción de la variable dependiente

\begin{tabular}{|c|c|c|c|}
\hline \multirow{4}{*}{$\begin{array}{l}\text { Independencia } \\
\text { económica }\end{array}$} & \multirow[b]{3}{*}{ Sí } & \multicolumn{2}{|c|}{ Independencia residencial } \\
\hline & & Sí & No \\
\hline & & Emancipación & Independencia económica \\
\hline & & $N=621$ & $N=267$ \\
\hline & No & $\begin{array}{l}\text { Independencia residencial } \\
\text { y dependencia económica }\end{array}$ & $\begin{array}{l}\text { Dependencia residencial } \\
\text { y económica }\end{array}$ \\
\hline & & $N=222$ & $N=865$ \\
\hline
\end{tabular}

Fuente: elaboración propia a partir de los datos del diseño del III Plan Integral de Juventud de Andalucía.

indican si los jóvenes siguen residiendo con sus padres y/o sus familiares o bien si se han marchado del hogar de sus progenitores para vivir solos o solos con hijos, con su pareja con o sin hijos o bien con personas no emparentadas, compartiendo vivienda. De esta forma, se han recodificado dichas categorías en dos: jóvenes independizados o no independizados residencialmente ${ }^{7}$.

En segundo lugar, se contempla la cuestión de la independencia económica por medio de la pregunta: «¿Cuál de las siguientes afirmaciones define mejor tu situación económica personal?». Las categorías de respuesta indican en qué medida los jóvenes son económicamente autónomos ${ }^{8}$. Así, se han recodificado estas categorías en dos: jóvenes dependientes o independientes económicamente?

De esta forma, se observa qué jóvenes se han emancipado, caracterizados por una independencia tanto económica como residencial, así como también los que son independientes residencialmente pero no económicamente, y viceversa. Finalmente, se recoge a los jóvenes que son totalmente dependientes.

\section{Variables independientes}

Con el fin de contrastar las hipótesis del estudio, se han utilizado una serie de variables sociodemográficas:

La edad, escogiendo tres intervalos que oscilan entre los 18 y los 35 años. El «primer tramo de edad» agrupa a los jóvenes de 18 a 24 años; el «segundo

7. Se considera a un joven como no independizado residencialmente aquel que vive con sus progenitores o con uno de ellos, así como también con otros familiares o parientes. Por otra parte, se considera como independizado residencialmente a aquel que vive solo con hijos, con su pareja (con o sin hijos) o con otra persona o personas no emparentadas.

8. Dicha variable se subdivide en las siguientes categorías: «vivo exclusivamente de mis ingresos», "vivo principalmente de mis ingresos, con la ayuda de otra persona o personas», "vivo principalmente de los ingresos de otra persona o personas, con algunos ingresos propios» $\mathrm{y}$ "vivo exclusivamente de los ingresos de otra persona o personas».

9. Se considera a un joven como independiente económicamente a aquel que vive exclusivamente o principalmente de sus ingresos, mientras que se considera dependiente económicamente a aquel que vive exclusivamente o principalmente de los ingresos de otra persona o personas. 
tramo de edad» hace referencia a los de 25 a 29 años, y, finalmente, el «tercer tramo de edad» se ocupa de los de 30 a 35 años.

El sexo, cuyas categorías son: "varón» y «mujer».

El nivel de estudios, introduciendo cada una de las categorías como variable dummy para su posterior análisis de regresión. Son las siguientes: «estudios primarios», "estudios secundarios» y "estudios superiores». Los "estudios primarios» contemplan a todos aquellos jóvenes que han finalizado su etapa formativa antes de terminar la educación secundaria obligatoria; es decir, que se encuentran en una de estas tres categorías: no saben leer ni escribir, tienen estudios primarios o no han completado la educación secundaria obligatoria. Por su parte, los «estudios secundarios» incorporan a aquellas categorías que corresponden a la finalización de los siguientes estudios: educación secundaria obligatoria, bachiller y grado medio (formación profesional). Finalmente, los «estudios superiores» contienen a aquellos jóvenes que tienen como mínimo uno de los siguientes niveles: grado superior (formación profesional), diplomatura, licenciatura o grado, máster y doctorado.

En el caso de los jóvenes que no están estudiando, se incorpora la intención de seguir estudiando como variable a analizar. Esta se divide en las categorías «tener intención de seguir estudiando" o "no tener intención de seguir estudiando».

La condición sociolaboral está construida a partir de una serie de categorías que forman parte de distintas variables. En primer lugar, se ha introducido una primera categoría que hace referencia al hecho de estar "ocupados». En segundo lugar, se tiene en cuenta a aquellos jóvenes que están «desempleados» $y$, finalmente, a aquellos que se encuentran dentro de un periodo formativo, por lo que adquieren la condición de «estudiantes».

Respecto al hecho de tener pareja estable como variable a analizar, se incorpora por medio de las categorías «tener pareja estable» o "no tener pareja estable».

Se integra la variable situación reproductiva a través de las categorías «tener hijos» o «no tener hijos».

En el caso de los jóvenes que no tienen hijos, se adhiere la intención de tener hijos como variable a analizar. Dicha variable se subdivide en las categorías «tener intención de tener hijos» o "no tener intención de tener hijos».

El tamaño del municipio señala su número de habitantes. Dicha variable se distribuye en las siguientes categorías: «municipio pequeño» (entre 0 y 10.000 habitantes), "municipio medio» (entre 10.001 y 50.000 habitantes) y "municipio grande» (desde 50.001 habitantes).

En la tabla 3 se observa, en primer lugar, que la edad muestra grandes diferencias porcentuales con respecto a cada una de las situaciones de independencia; cuestión evidente, puesto que la relación entre emancipación y edad está más que probada, por lo que no se incorpora esta variable al modelo de regresión posterior ${ }^{10}$. Con respecto al sexo, destaca la independencia económica

10. Otra cuestión por la que no resulta pertinente incorporar la variable edad al análisis se debe a la posibilidad de que existan problemas derivados de una posible colinealidad alta entre variables, como podría ser el caso de la edad y el nivel de estudios finalizado o tener hijos, entre otras. 
Tabla 3. Características sociodemográficas de las distintas formas de independencia, en función de la situación económica y residencial en los jóvenes de 18 a 35 años (\%)

\begin{tabular}{|c|c|c|c|c|c|}
\hline & & $\begin{array}{l}\text { Emancipación } \\
\text { (independencia } \\
\text { residencial y } \\
\text { económica) }\end{array}$ & $\begin{array}{l}\text { Independencia } \\
\text { económica y } \\
\text { dependencia } \\
\text { residencial }\end{array}$ & $\begin{array}{l}\text { Independencia } \\
\text { residencial y } \\
\text { dependencia } \\
\text { económica }\end{array}$ & $\begin{array}{c}\text { Dependencia } \\
\text { residencial y } \\
\text { económica }\end{array}$ \\
\hline $\begin{array}{l}\text { Porcentaje sobre el } \\
\text { total de la muestra }\end{array}$ & & 31,65 & 13,48 & 11,21 & 43,66 \\
\hline \multirow[t]{3}{*}{ Tramo de edad 1} & 18-23 años & 4,40 & 12,36 & 11,94 & 71,27 \\
\hline & 24-29 años & 32,97 & 20,58 & 16,40 & 30,03 \\
\hline & 30-35 años & 66,78 & 12,43 & 10,05 & 10,73 \\
\hline \multirow[t]{2}{*}{$\operatorname{Sexo}^{2}$} & Varón & 53,90 & 57,30 & 25,22 & 53,41 \\
\hline & Mujer & 46,09 & 42,69 & 74,77 & 46,58 \\
\hline \multirow[t]{3}{*}{ Nivel de estudios ${ }^{2}$} & Estudios primarios & 11,83 & 21,75 & 14,86 & 28,40 \\
\hline & Estudios secundarios & 34,83 & 38,93 & 62,61 & 56,69 \\
\hline & Estudios superiores & 53,33 & 39,31 & 22,52 & 14,90 \\
\hline \multirow{2}{*}{$\begin{array}{l}\text { Intención de seguir } \\
\text { estudiando }{ }^{1}\end{array}$} & Sí & 27,44 & 22,32 & 6,97 & 43,25 \\
\hline & No & 48,21 & 15,89 & 15,89 & 20,00 \\
\hline \multirow{3}{*}{$\begin{array}{l}\text { Condición } \\
\text { sociolaboral }^{1}\end{array}$} & Ocupados & 61,32 & 18,16 & 8,71 & 11,82 \\
\hline & Desempleados & 11,94 & 18,66 & 20,15 & 49,25 \\
\hline & Estudiantes & 1,35 & 2,24 & 10,76 & 85,65 \\
\hline \multirow{2}{*}{$\begin{array}{l}\text { Tener pareja } \\
\text { estable }^{1}\end{array}$} & Sí & 40,98 & 12,55 & 15,86 & 30,59 \\
\hline & No & 20,67 & 14,51 & 6,27 & 58,53 \\
\hline \multirow[t]{2}{*}{ Tener hijos $^{1}$} & Sí & 60,22 & 7,72 & 21,13 & 10,90 \\
\hline & No & 23,67 & 15,23 & 8,43 & 52,64 \\
\hline \multirow{2}{*}{$\begin{array}{l}\text { Intención de } \\
\text { tener hijos }\end{array}$} & Sí & 27,44 & 22,32 & 6,97 & 43,25 \\
\hline & No & 48,21 & 15,89 & 17,81 & 20,00 \\
\hline \multirow[t]{3}{*}{ Municipio² } & Municipio pequeño & 26,70 & 18,84 & 10,99 & 43,45 \\
\hline & Municipio medio & 32,99 & 11,16 & 10,82 & 44,83 \\
\hline & Municipio grande & 32,63 & 12,79 & 11,50 & 43,05 \\
\hline
\end{tabular}

Notas

1. Porcentajes en filas (todos los valores de la fila suman 100).

2. Porcentajes en columnas (todos los valores de la columna suman 100).

Fuente: elaboración propia a partir de los datos de la encuesta del Diseño del III Plan Integral de Juventud de Andalucía.

y no residencial de los varones, así como también la independencia residencial y no económica de las mujeres.

El nivel de estudios refleja diferencias significativas con respecto a la situación residencial y económica. Se observa que, a mayor nivel de estudios, más alta es la probabilidad de que los jóvenes estén emancipados. Por su parte, la intención de seguir estudiando se relaciona en mayor medida con unos mayores indicadores de dependencia. 
Con respecto a la condición sociolaboral, destaca que ser estudiante obtiene los mayores porcentajes de dependencia, mientras que estar ocupado está estrechamente relacionado con el hecho de estar emancipado.

Tener pareja estable o hijos obtiene porcentajes más altos cuando se trata de situaciones de independencia, tanto residencial como económica, sobre todo en el caso de la descendencia.

Finalmente, con respecto al tamaño del municipio, no se observan diferencias porcentuales significativas, por lo que tampoco se va a incluir dicha variable en el análisis. Cabe mencionar que el coeficiente de la ji al cuadrado en cada cruce de variables ha señalado una asociación estadística significativa entre la variable dependiente y cada una de las variables independientes, a excepción del tamaño del municipio.

\section{Resultados}

En esta sección, en primer lugar, se exponen los resultados obtenidos mediante una regresión logística multinomial (tabla 4). Con ello se pretende conocer la probabilidad de que ocurra el fenómeno señalado por la variable dependiente, frente a la probabilidad de que no ocurra. Se exponen los descriptivos de ajuste básicos al respecto, así como también los resultados obtenidos a partir de ellos. La categoría de la variable dependiente omitida en el análisis es la de «dependencia residencial y económica», por tanto, la que se encuentra opuesta a la emancipación completa.

Puesto que, a primera vista, se observa que todas las variables independientes introducidas en el análisis influyen en la probabilidad de emanciparse o de ser independiente económicamente o residencialmente, se acepta la hipótesis $\mathrm{H} 1$.

\subsection{Jóvenes emancipados: independencia económica y residencial}

La primera categoría del modelo analizado tiene como variable dependiente a los jóvenes que se han independizado tanto económicamente como residencialmente, a los que mencionamos como emancipados. Respecto a los resultados que aparecen como significativos, en primer lugar, respecto la variable sexo, se observa que el hecho de ser varón disminuye la posibilidad de emanciparse; sin embargo, la relación entre variables no es significativa. Por tanto, el sexo en un principio no está relacionado con la decisión emancipatoria.

En segundo lugar, los jóvenes con estudios superiores configuran una categoría que está relacionada con una mayor probabilidad de emanciparse. Además, la categoría que incorpora a los que ya finalizaron su etapa formativa, aunque tienen intención de seguir estudiando, está altamente relacionada con el hecho de no estar emancipados. Por tanto, en principio se rechaza una parte de la hipótesis H1.1 (que indica que el sexo no está relacionado con la decisión emancipatoria) y aceptamos otra (la relación existente entre el nivel de estudios y la emancipación). Sin embargo, con respecto al sexo se observa a continua- 
Tabla 4. Modelo para jóvenes de 18 a 35 años independizados residencialmente, económicamente o ambos

\begin{tabular}{|c|c|c|c|c|c|c|}
\hline & \multicolumn{2}{|c|}{ Emancipación } & \multicolumn{2}{|c|}{$\begin{array}{l}\text { Independencia } \\
\text { económica }\end{array}$} & \multicolumn{2}{|c|}{$\begin{array}{l}\text { Independencia } \\
\text { residencial }\end{array}$} \\
\hline & $\operatorname{Exp}(2)$ & $\begin{array}{l}\text { Error } \\
\text { estándar }\end{array}$ & $\operatorname{Exp}(2)$ & $\begin{array}{l}\text { Error } \\
\text { estándar }\end{array}$ & $\operatorname{Exp}(2)$ & $\begin{array}{c}\text { Error } \\
\text { estándar }\end{array}$ \\
\hline \multicolumn{7}{|l|}{ Sexo } \\
\hline Varón & $-0,366$ & 0,130 & $-0,082$ & 0,731 & $-1,389^{\star \star \star}$ & 0,000 \\
\hline Mujer & - & - & - & - & - & - \\
\hline \multicolumn{7}{|l|}{ Nivel de estudios } \\
\hline Estudios primarios & 0,208 & 0,569 & $0,714^{\star *}$ & 0,017 & $-0,485$ & 0,249 \\
\hline Estudios secundarios & - & - & - & - & - & - \\
\hline Estudios superiores & $1,018^{\star \star \star}$ & 0,000 & $0,778^{\star \star \star}$ & 0,005 & $0,727^{\star \star}$ & 0,023 \\
\hline $\begin{array}{l}\text { Intención de seguir } \\
\text { estudiando }\end{array}$ & $-0,934^{\star \star \star}$ & 0,001 & $-0,118$ & 0,658 & $-1,053^{\star \star \star}$ & 0,004 \\
\hline \multicolumn{7}{|l|}{$\begin{array}{l}\text { Condición } \\
\text { sociolaboral }\end{array}$} \\
\hline Ocupados & $2,732^{\star \star \star}$ & 0,000 & $1,235^{\star \star \star}$ & 0,000 & 0,162 & 0,592 \\
\hline Desempleados & - & - & - & - & - & - \\
\hline Estudiantes & $-11,250^{\star \star *}$ & 0,000 & $-13,798^{\star \star \star}$ & 0,000 & $-11,810^{\star \star *}$ & 0,000 \\
\hline Tener pareja estable & $0,870^{\star \star \star}$ & 0,000 & 0,087 & 0,691 & $1,782^{\star \star \star}$ & 0,000 \\
\hline No tener pareja & - & - & - & - & - & - \\
\hline Tener hijos & $3,157^{\star \star \star}$ & 0,000 & $1,588^{\star \star \star}$ & 0,000 & $2,935^{\star \star \star}$ & 0,000 \\
\hline No tener hijos & - & - & - & - & - & - \\
\hline Intención de tener hijos & $0,433^{*}$ & 0,081 & $-0,078$ & 0,748 & $0,730^{* *}$ & 0,036 \\
\hline Sin intención de tener hijos & - & - & - & - & - & - \\
\hline \multicolumn{7}{|l|}{$N=816$} \\
\hline \multicolumn{7}{|l|}{ Pseudo R2 = 0,2233 } \\
\hline P-valor: ${ }^{* * *} p<0,01,{ }^{* *} p<0,0$ & $05,{ }^{*} p<0,1$ & & & & & \\
\hline
\end{tabular}

ción que, en referencia a la independencia residencial, sí existe relación. Por tanto, aceptamos la hipótesis H1.1.

Respecto a la condición sociolaboral de los jóvenes, se advierte que la situación de estudiante mantiene una relación negativa con el hecho de estar emancipado y se posterga el abandono del hogar. Ocurre lo contrario con los jóvenes empleados: se acercan más a la probabilidad de emanciparse.

Finalmente, tener pareja se asocia con el hecho de estar emancipado. Esto ocurre también con los jóvenes que dicen tener hijos, aunque esta segunda variable establece una relación más fuerte respecto a la situación familiar. Por tanto, convertirse en padre o en madre está estrechamente relacionado con el hecho de abandonar el hogar de los progenitores. Respecto a la relación entre el deseo o la intención de tener hijos, se observa que esta circunstancia se vincula al hecho de estar emancipados. Se puede afirmar 
que la intención de tener descendencia implica una mayor probabilidad de emanciparse.

De esta forma, aceptamos la hipótesis H1.2: estar ocupado, tener pareja, hijos y/o tener intención de ello son circunstancias que favorecen la emancipación de los jóvenes. Por el contrario, cuestiones como la intención de seguir formándose en algún momento, así como encontrarse dentro de la categoría de estudiante o estar desempleado desalienta la emancipación, por lo que se acepta la hipótesis H1.3.

Finalmente, se acepta la hipótesis $\mathrm{H} 2$, la cual indica que ciertas variables que influyen o no en la emancipación de los jóvenes cambian, tanto en lo que se refiere al nivel de asociación como a la dirección y/o a la fuerza, al tener en cuenta, por un lado, la emancipación completa y, por otro, la independencia económica y no residencial, así como también la independencia residencial y no económica. A continuación se explican dichos cambios y se detallan estas nuevas relaciones.

\subsection{Jóvenes independizados económicamente, no residencialmente}

Con respecto a la segunda categoría analizada de la variable dependiente, esta concierne a los jóvenes que están independizados económicamente, aunque no residencialmente. Se observan menos relaciones estadísticamente significativas entre las variables y las categorías analizadas como variables independientes que en el modelo anterior.

Por su parte, la variable nivel de estudios muestra cierta relación con el hecho de ser económicamente independiente, tanto en la categoría de «estudios primarios» como de «estudios superiores».

Tal y como ocurre en el modelo anterior, la categoría de jóvenes estudiantes, dentro de la condición sociolaboral, se relaciona estrechamente con el hecho de no ser independientes económicamente. Por otra parte, estar trabajando se relaciona igualmente con una mayor posibilidad de ser independiente económicamente, aunque no residencialmente.

Nuevamente, tener hijos acerca más a los jóvenes a ser independientes económicamente, aunque con bastante menor intensidad que en la independencia completa.

El sexo, la intención de seguir estudiando, tener pareja estable y la intención de tener hijos son variables que no muestran una relación significativa con la probabilidad de ser una joven o un joven independizado económicamente, aunque no residencialmente.

\subsection{Jóvenes independizados residencialmente, no económicamente}

Para concluir, la tercera categoría a analizar de la variable dependiente se refiere a los jóvenes que están independizados residencialmente, aunque no económicamente. Aparecen más relaciones estadísticamente significativas entre las variables y las categorías analizadas que en la anterior categoría estudiada, con 
algunas divergencias en su comparación con los dos modelos anteriormente expuestos.

En esta ocasión, el sexo está estrechamente relacionado con la variable dependiente. Concretamente, ser varón conlleva una menor probabilidad de ser independiente residencialmente, aunque no económicamente.

En lo que al nivel de estudios concierne, únicamente la categoría «estudios superiores» tiene una relación estadísticamente significativa con la variable dependiente. El hecho de haber llegado a dicho nivel académico aumenta la probabilidad de ser independiente residencialmente y no económicamente. En esta ocasión, tal y como ocurre en el primer modelo, la intención de seguir estudiando también establece una fuerte relación con la variable independiente. Dicha pretensión implica una menor probabilidad de encontrarse en un estatus de independencia residencial.

Concluyendo, el hecho de tener pareja estable, hijos y mostrar intención de tenerlos se relaciona fuertemente con ser independientes residencialmente, aunque no económicamente. Al llevar a cabo un análisis descriptivo, se observa que este hecho se debe a un porcentaje considerable de mujeres que conviven con sus parejas y que son estas las que llevan el peso económico del hogar.

Cabe señalar que se ha comprobado que el tamaño del municipio no influye en la decisión emancipatoria (en este caso, en ninguna de las tres categorías de la variable dependiente), de forma que se ha excluido del modelo para obtener mayor calidad de resultados.

\subsection{Efectos marginales}

Los efectos marginales que se muestran en la tabla 5 permiten conocer los factores estructurales (variables demográficas y socioeconómicas) de los jóvenes de la muestra que aumentan o disminuyen la probabilidad de que un joven sea independiente de forma completa o no (solo económicamente o residencialmente), así como también la exactitud porcentual de esa aproximación o lejanía con respecto a la variable dependiente.

$\mathrm{Al}$ analizar detenidamente cada una de las variables de interés para este estudio, en primer lugar, en la tabla 5 se observa a grosso modo que todos los factores estructurales analizados afectan de forma distinta al hecho de estar completamente emancipados, ser independientes solo económicamente o bien únicamente de forma residencial. Además, las relaciones que se establecen también difieren en función de la situación de independencia que tenga el joven en cuestión.

Concretamente, las diferencias significativas a mencionar entre las distintas categorías analizadas que contiene la variable dependiente, muestran que, atendiendo al sexo de los jóvenes encuestados, pese a que no aparecen diferencias significativas en lo que respecta a la emancipación completa, son las mujeres las que tienen una ligera mayor probabilidad de encontrarse dentro de esta categoría.

Por otra parte, el hecho de ser varón aumenta la probabilidad de ser económicamente autosuficiente y no residencialmente, mientras que ocurre 
Tabla 5. Efectos marginales medios de las variables independientes utilizadas para los modelos

\begin{tabular}{|c|c|c|c|}
\hline $\begin{array}{c}\text { Variables } \\
\text { independientes }\end{array}$ & Emancipación & $\begin{array}{l}\text { Independencia } \\
\text { económica }\end{array}$ & $\begin{array}{l}\text { Independencia } \\
\text { residencial }\end{array}$ \\
\hline \multicolumn{4}{|c|}{ Sexo (categoría de referencia: mujer) } \\
\hline Varón & $-0,3 \%$ & $6,9 \%$ * & 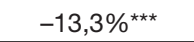 \\
\hline \multicolumn{4}{|c|}{ Nivel de estudios (categoría de referencia: estudios secundarios) } \\
\hline Estudios primarios & $-2,1 \%$ & $13,8 \%$ ** & $-7,0 \%$ *** \\
\hline Estudios superiores & $12,2 \%$ *** & $1,0 \%$ & $-0,1 \%$ \\
\hline Intención de seguir estudiando & 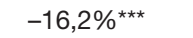 & $10,8 \%$ ** & $-5,6 \%$ ** \\
\hline \multicolumn{4}{|c|}{ Condición sociolaboral (categoría de referencia: sector servicios y trabajadores manuales) } \\
\hline Ocupados & $44,9 \%{ }^{* * *}$ & $-0,7 \%$ & $-14,9 \%$ *** \\
\hline Estudiantes & $-42,4 \%$ *** & $-26,6 \%$ *** & $-12,1 \%$ *** \\
\hline \multicolumn{4}{|c|}{ Situación sentimental (categoría de referencia: no tener pareja estable) } \\
\hline Tener pareja estable & $12,2 \%{ }^{\star \star *}$ & $-12,9 \%$ *** & $12,2 \%$ *** \\
\hline \multicolumn{4}{|c|}{ Situación reproductiva (categoría de referencia: no tener hijos) } \\
\hline Tener hijos & $36,7 \% \%^{* \star *}$ & $-15,5 \%$ *** & $6,9 \%$ ** \\
\hline Intención de tener hijos & $8,0 \%$ * & $-8,7 \%$ ** & $5,4 \%$ ** \\
\hline
\end{tabular}

Significación: * Significativa al 90\%, ${ }^{\star \star}$ Significativa al 95\%, ${ }^{\star \star \star}$ Significativa al 99\%. P-valores entre paréntesis.

Para llevar a cabo este análisis, se ha tomado como punto de referencia la cuarta categoría (base), es decir, los jóvenes completamente dependientes (económicamente y residencialmente).

Fuente: elaboración propia a partir de los datos de la encuesta del Diseño del III Plan Integral de Juventud de Andalucía.

una tendencia contraria cuando se trata de independencia residencial, en los casos en los que la independencia no es completa. Esta diferencia sustancial se puede atribuir, entre otras causas, al hecho de que, en los casos de parejas jóvenes que conviven en un mismo hogar, sigue siendo el varón el sustentador principal. Las mujeres se lanzan a la formación de un nuevo hogar a pesar de no ser plenamente autosuficientes, mientras que los varones tienden a mantenerse algo más en el hogar de sus progenitores a pesar de tener independencia económica.

En definitiva, este hecho se puede atribuir a que las mujeres se emancipan generalmente al formar una pareja, mientras que los varones, si no tienen cierta independencia económica, no abandonan el hogar de los progenitores.

En cuanto al nivel de estudios, se observa que, dentro del grupo de aquellos jóvenes que gozan de una emancipación completa, la mayor probabilidad de encontrarse en dicha categoría va de la mano de tener un nivel de estudios superiores, seguida por los jóvenes que han terminado los estudios secundarios, aunque muy de lejos.

Por otra parte, los jóvenes con estudios primarios son los que muestran una mayor probabilidad de gozar de independencia económica, aunque no residencial, seguidos de los que tienen estudios superiores. Muchos de los jóvenes con estudios primarios empezaron a formar parte de la condición de empleados 
muy tempranamente ${ }^{11}$, por lo que cuentan con recursos económicos suficientes como para cubrir sus gastos de forma prácticamente autónoma. Por otra parte, la menor probabilidad de que se encuentren dentro de esta categoría los jóvenes con estudios secundarios se puede explicar, entre otras causas, por el hecho de que muchos de ellos todavía son estudiantes, y, como se ha observado, dicha categoría está estrechamente relacionada con el hecho de ser dependientes, tanto residencial como económicamente.

Finalmente, son los jóvenes con estudios secundarios los que tienen mayor propensión a ser independientes residencialmente, no económicamente. Los que tienen estudios primarios, concretamente, presentan un $7 \%$ menos de probabilidades de encontrarse dentro de este tipo de independencia. Como ya hemos visto antes, tienden más a relacionarse con una mayor probabilidad de estar completamente emancipados.

En definitiva, está claro que prolongar los estudios y la formación implica no obtener recursos suficientes para conseguir cierta independencia económica. Los jóvenes que han dejado de estudiar y están trabajando en muchas ocasiones perciben una baja remuneración, lo que les impide emanciparse residencialmente.

De hecho, aquellos jóvenes que manifiestan su intención de seguir estudiando tienen una menor probabilidad de estar emancipados completamente. Con respecto a una independencia incompleta, aquellos que dicen tener intención de seguir estudiando es más probable que sean independientes económicamente, aunque no residencialmente. En muchos casos, estando ocupados, pretenden obtener un empleo de mayor calidad, una retribución más elevada y mejores condiciones laborales (para conseguir una emancipación completa, entre otras cosas); por tanto, tienen el deseo de proseguir con su formación en un futuro. Cruzando los datos disponibles, efectivamente se observa cómo un $54,1 \%$ de los jóvenes con intención de seguir estudiando están ocupados, mientras que un $44,4 \%$ de ellos están desempleados (el porcentaje restante pertenece a los que se encuentran en condición de estudiantes).

En cambio, aquellos jóvenes que son independientes residencialmente, aunque no económicamente, se relacionan negativamente con la intención de seguir estudiando. Dicho de otra forma, aquellos que tienen intención de seguir estudiando muestran una menor probabilidad de encontrarse independizados residencialmente, aunque no económicamente. Esto refleja que hay muchos casos de jóvenes que, una vez abandonada o finalizada su etapa formativa, pretenden retomarla habitando en la vivienda de sus progenitores. En ocasiones no se marchan del hogar familiar en ningún momento, mientras que otros regresan a él tras haberse independizado residencialmente en al

11. Estamos tratando con jóvenes de 18 o más años de edad. Por tanto, aquellos que únicamente tienen un nivel de estudios primarios han dejado de estudiar hace ya algunos años (no se encuentran todavía en condición de estudiantes, excepto aquellos casos en los que se han retomado los estudios, porcentaje de jóvenes a considerar, pero no determinante). Por tanto, se puede atribuir a dicha explicación. 
menos una ocasión (jóvenes boomerang). Y es que, para disponer de tiempo para estudiar, no pueden dedicar mucho a actividades retributivas, por lo que difícilmente pueden alcanzar la emancipación completa en ese momento.

En cuanto a la condición sociolaboral, cabe afirmar que aquellos jóvenes que están laboralmente ocupados presentan una mayor probabilidad de ser independientes económica y residencialmente, mientras que los estudiantes muestran una tendencia inversa. Las diferencias entre ambas circunstancias son muy considerables.

Por su parte, los jóvenes que están desempleados tienen más probabilidad tanto de ser independientes residencialmente y no económicamente, como de ser independientes económicamente y no residencialmente. Esto se debe a que el hecho de estar ocupado está totalmente relacionado con la independencia absoluta (económica y residencial), como ya hemos visto, no con solo una de ellas. Por otra parte, el hecho de ser estudiante está absolutamente vinculado con la dependencia completa (no hay ningún tipo de independencia teniendo esta condición).

Seguidamente, respecto a la circunstancia de tener pareja estable, se observa que dicha condición está relacionada con una mayor probabilidad de estar emancipado completamente. De hecho, tener pareja estable es una de las variables que explica una mayor probabilidad de estar emancipado.

La pareja estable se convierte en una fuente de apoyo económico y, por esta razón, implica una menor probabilidad de ser económicamente autosuficiente, pero no independiente residencialmente. Este hecho se puede atribuir a que, en los casos donde las parejas estables conviven en el mismo hogar, los gastos con los que cuentan se comparten entre ambos miembros, por lo que no pueden ser considerados independientes económicamente según la consideración llevada a cabo para su medición en este artículo. La subida del precio de la vivienda en los últimos años, así como también el descenso de los salarios, entre otras causas, implica que con un único sueldo no se puedan hacer cargo de los gastos únicamente uno de los dos miembros de la pareja.

Por otro lado, los jóvenes con pareja estable están relacionados con una mayor probabilidad de ser independientes residencialmente, aunque no económicamente. Como hemos dicho, muchos de ellos conviven con sus parejas, por lo que se consideran como independientes en lo que respecta a la residencia por haber abandonado el hogar de sus progenitores para formar uno propio.

La variable tener hijos también está estrechamente relacionada con el hecho de que un joven esté completamente emancipado, es decir, haya formado un hogar propio y disponga de ingresos que le permitan ser autosuficiente económicamente.

Con respecto a la independencia incompleta, se observa que, por un lado, tener hijos implica una menor probabilidad de ser independiente económicamente, aunque sí residencialmente. Asumir el coste de uno o varios hijos una persona sola es una tarea compleja, y aquí queda evidente el hecho de que se relaciona con la colaboración de otras personas, normalmente la pareja, para asumir los gastos. Por tanto, considerando que la independencia económica 
supone cubrir los gastos propios de forma prácticamente autónoma, queda evidente la situación en esta categoría de la variable dependiente por parte de los jóvenes que tienen descendencia.

Por otro lado, se observa que tener hijos se relaciona con una mayor probabilidad de ser independientes residencialmente, aunque no económicamente. En definitiva, queda claro que el hecho de tener hijos se relaciona positivamente con la formación de un hogar propio y la consecuente independencia residencial. Sin embargo, no ocurre lo mismo con la independencia económica, ya que se necesita de otros ingresos para afrontar dichos gastos.

Finalmente, y para concluir, la intención de tener hijos también proporciona información a considerar con respecto al tipo de independencia, puesto que implica una mayor probabilidad de encontrarse en una situación de emancipación completa; por otra parte, dicha intención se relaciona con una menor probabilidad de ser independiente económicamente y una mayor probabilidad de ser independiente residencialmente.

\section{Conclusiones}

El objetivo primordial de esta investigación consiste en conocer cuáles son los factores que influyen o determinan en mayor medida el hecho de que un joven se emancipe en Andalucía. Como novedad, se ha distinguido dentro de la emancipación qué jóvenes son independientes residencialmente y/o económicamente para, de esta forma, averiguar si existen diferencias en los factores que influyen en que un joven sea independiente residencialmente y no económicamente, o viceversa. Otra cuestión a tener en cuenta radica en el hecho de que se está analizando una base de datos de la juventud andaluza actual (último trimestre de 2017), todavía no explotada y utilizada para llevar a cabo el diseño del III Plan Integral de Juventud de Andalucía.

Respecto a las hipótesis planteadas, en primer lugar, se comprueba que las variables independientes utilizadas afectan a la emancipación de los jóvenes. En referencia al sexo, este está relacionado con la decisión emancipatoria, como señalan Mitchell et al. (2017), Colom y Molés (2016) o Bellart y Oller (2005). El hecho de ser varón disminuye la probabilidad de emanciparse completamente, así como también de ser independiente residencialmente. Sin embargo, estos tienen más probabilidad de ser independientes económicamente, aunque no residencialmente.

Se observa que los jóvenes con estudios superiores tienen mayor probabilidad de gozar de una independencia completa. Por otra parte, los estudios primarios disminuyen la probabilidad de ser residencialmente independiente, aunque no ocurre lo mismo cuando se trata de ser solo económicamente independiente. Con respecto a los jóvenes que muestran la intención de seguir estudiando, dicha categoría está relacionada con el hecho de no estar emancipado o de no tener algún tipo de independencia.

Estar ocupado favorece la emancipación de los jóvenes, tal y como señalan Iacovou (2010), Ghidoni (2002), Colom y Molés (2016), Bellart y Oller 
(2005) y Moreno (2012). Por otra parte, estar estudiando se relaciona negativamente con el hecho de ser completamente independiente (Miret, 2006; Jones, 1995; Colom y Molés, 2016; Bellart y Oller, 2005; Ballesteros et al., 2012; South y Lei, 2015), por lo que el hecho de estar estudiando se vincula con la dependencia completa. Se sigue manteniendo la relación positiva entre empleo e independencia económica, aunque no residencial, mientras que la condición de estudiante lo hace a la inversa. Cabe mencionar que los jóvenes desempleados son los que tienen una mayor probabilidad tanto de ser independientes residencialmente y no económicamente, como de ser independientes económicamente y no residencialmente.

El hecho de tener una pareja estable e hijos, así como también la intención de tenerlos, fomenta que los jóvenes abandonen el hogar de sus progenitores. Concretamente, tener pareja se asocia con la circunstancia de estar emancipados, tal y como señalan Huinink y Konietzka (2000) y South y Lei (2015). Además, tal y como indican Goldscheider et al. (2014) y Holdsworth et al. (2002), convertirse en padre o madre está estrechamente relacionado con el hecho de abandonar el hogar de los progenitores. Respecto a la intención de tener hijos, también implica una mayor probabilidad de emanciparse. Sin embargo, tener pareja estable implica una menor probabilidad de ser económicamente autosuficiente, aunque no residencialmente. Esto mismo ocurre con el hecho de tener hijos, lo que se relaciona con una mayor probabilidad de ser independientes residencialmente, aunque no económicamente. En definitiva, queda claro que tener hijos se relaciona positivamente con la formación de un hogar propio y la consecuente independencia residencial. Sin embargo, no ocurre lo mismo con la independencia económica, ya que se necesita de otros ingresos para afrontar dichos gastos. Finalmente, la intención de tener descendencia implica una mayor probabilidad de encontrarse en una situación de emancipación completa; por otra parte, dicha intención se relaciona con una menor probabilidad de ser independiente económicamente y con una mayor probabilidad de ser independiente residencialmente.

Con respecto al tamaño del municipio de residencia, se ha observado en los inicios del análisis que no tiene ningún tipo de relación con el hecho de estar emancipado ni con otro tipo de independencia incompleta.

De esta forma, las variables que influyen en la emancipación de los jóvenes son divergentes, al tener en cuenta, por un lado, la emancipación completa y, por otro, la independencia económica y no residencial, así como también la independencia residencial y no económica.

En definitiva, se observa que los jóvenes que se aproximan más a la finalización de la transición de la juventud (empleados, con estudios superiores finalizados y con hijos, entre otras categorías) tienen más probabilidades de haber formado un hogar propio y de ser autosuficientes económicamente. Sin embargo, aquellos jóvenes que todavía están formándose o tienen intención de retomar los estudios, están desempleados y no tienen descendencia ni intención de tenerla, entre otras cuestiones, se aproximan más a formas de independencia incompletas (residencial o económica), o bien nulas. 
Concretamente, aquellas variables de mayor interés en Andalucía (empleo y nivel de estudios), las cuales se relacionan positivamente con una independencia completa (estar empleado y tener un nivel de estudios superiores), pueden predecir que emanciparse en dicha comunidad autónoma resulta más difícil que en el resto de España. Sin embargo, habría que investigar acerca del papel de las demás variables en la emancipación andaluza (tener pareja estable o hijos, por ejemplo), puesto que las motivaciones que llevan a que un joven se emancipe pueden ser distintas entre comunidades. Ya sabemos que sí lo son entre ciertos países de Europa por ejemplo: en los países del sur de nuestro continente la emancipación está más relacionada con la unión en pareja-, por lo que sería de gran utilidad enfocar próximamente hacia esa dirección la continuación del presente estudio.

En un futuro sería interesante profundizar en las explicaciones acerca de por qué las variables independientes no tienen la misma relación con cada una de las categorías de la variable dependiente. Algunas de ellas se han podido contrastar mediante los datos disponibles, mientras que otras no, por falta de información en la encuesta.

\section{Agradecimientos}

Se agradece la colaboración y el apoyo recibido de Jorge Guardiola con respecto a la calidad del artículo.

Los posibles errores contenidos en este artículo son responsabilidad exclusiva de los autores.

\section{Referencias bibliográficas}

Aasve, A.; Burgess, S.; Chesher, A. y Propper, C. (2002). «Transition from Home to Marriage of Young Americans». Journal of Applied Econometrics, 17, 1-23. $<$ https://doi.org/10.1002/jae.636>

Albertini, M. (2010). «La ayuda de los padres españoles a los jóvenes adultos: El familismo español en perspectiva comparada». En: Moreno, A. (eds.). «Juventud y familia desde una perspectiva comparada europea». Revista de Estudios de Juventud, 90.

Avery, R.; Goldscheider, F. y Speare, A.S. (1992). «Feathered Nest / Gilded Cage: Parental Income and Leaving Home in the Transition to Adulthood». Demography, 29(3), 375-388. $<$ https://doi.org/10.2307/2061824>

Ballesteros, J.C.; Megías, I. y Rodríguez, E. (2012). «Jóvenes y emancipación en España». Fundación de Ayuda contra la Drogadicción, 1-140.

Becker, S.O.; Bentolila, S.; Fernandes, A. e Ichino, A. (2010). «Youth emancipation and perceived job insecurity of parents and children». Journal of Population Economics, 23(3), 1047-1071. <https://doi.org/10.1007/s00148-008-0224-5>

Bellart, C. y Oller, J. (2005). «El acceso de los jóvenes a la vivienda: Una cuestión todavía no resuelta». Documentación Social, 138, 191-206. 
Bernardi, F. (2007). «Movilidad social y dinámicas familiares: Una aplicación al estudio de la emancipación familiar en España». Revista Internacional de Sociologia, 48, 33-54.

Billari, F.C. y Liefbroer, A.C. (2007). «Should I Stay or Should I Go?: The Impact of Age Norms on Leaving Home». Demography, 44(1), 181-198. <https://doi.org/10.1353/dem.2007.0000>

Blaauboer, M. y Mulder, C.H. (2010). "Gender differences in the impact of family background on leaving the parental home». Journal of Housing and the Built Environment, 25(1), 53-71. <https://doi.org/10.1007/s10901-009-9166-9>

Calvo, E. (2002). «Emancipación tardía y estrategia familiar». Estudios de Juventud, 58/2, 1-9. Recuperado de <http://www.injuve.es/sites/default/files/articulo1.pdf>.

Consejo de la Juventud de España (2015). Observatorio de Emancipación no 9 (Primer trimestre de 2015). Recuperado de <http://www.cje.org/es/publicaciones/ novedades/observatorio-de-emancipacion-n-9-primer-trimestre-2015/>.

- (2016). Observatorio de Emancipación no 12 (Primer semestre de 2016). Recuperado de <http://www.cje.org/es/publicaciones/novedades/observatorio-emancipacionprimer-semestre-2016/>.

- (2017). Observatorio de Emancipación no 14 (Primer semestre de 2017). Recuperado de <http://www.cje.org/es/publicaciones/novedades/observatorio-emancipacionprimer-semestre-2017/>.

Colom, M.C. y Molés, M.C. (2016). «Emancipación familiar en España: Análisis del comportamiento de los jóvenes en 1990, 2000 y 2010». Revista de Métodos Cuantitativos para la Economía y la Empresa, 22, 120-138.

Devolder, D. y Tejada, M.M. (2007). «Evolución reciente de la infecundidad y la fecundidad total: España en el contexto europeo». En: La constitución familiar en España. Madrid: Fundación BBVA, 139-198.

Ermisch, J. (1999). «Prices, Parents, and Young People’s Household Formation». Journal of Urban Economics, 45(1), 47-71. <https://doi.org/10.1006/JUEC.1998.2083>

Esping-Andersen, G. (1999). Social foundations of postindustrial economies. Oxford University Press. <https://doi.org/10.1093/0198742002.001.0001>

Gentile, Alessandro (2010). "De vuelta al nido en tiempos de crisis: Los boomerang kids españoles». Revista de Estudios de Juventud, 90, 181-203.

Ghidoni, M. (2002). «Determinants of young Europeans' decision to leave the parental household». University College of London. Working paper.

Goldscheider, F. y Goldscheider, C. (1999). The changing transition to adulthood: Leaving and returning home. Thousand Oaks, CA: Sage Publications.

Goldscheider, F.K.; Hofferth, S.L. y Curtin, S.C. (2014). «Parenthood and Leaving Home in Young Adulthood». Population Research and Policy Review, 33(6), 771-796. <https://doi.org/10.1007/s11113-014-9334-9>

Holdsworth, C.; Voas, D. y Tranmer, M. (2002). «Leaving home in Spain: When, where and why?». Regional Studies, 36(9), 989-1004. <https://doi.org/10.1080/0034340022000022206>

Huinink, J. y Konietzka, D. (2000). «Leaving Parental Home in the Federal Republic of Germany and the GDR: The changing interrelation of leaving home and other transition events to adulthood». Ponencia presentada en las jornadas Leaving Home: A European Focus. Max Planck Institute for Demographic Research Rostock. 
Iacovou, M. (2010). «Leaving Home: Independence, togetherness and income in Europe». Advances in Life Course Research, 15(4), 147-160. <https://doi.org/10.1016/j.alcr.2010.10.004>

INE, Instituto Nacional de Estadística (2018). «Población de 16 y más años por nivel de formación alcanzado, sexo y comunidad autónoma». Porcentajes respecto al total de cada comunidad. Recuperado de <http://www.ine.es/jaxiT3/Datos. htm? $\mathrm{t}=6369>$.

Jones, H. (1995). Leaving home. Buckingham: Open University Press.

Lesthaeghe, R. y Van De KaA, D.J. (1986). "Twee demografische transities». Bevolking: Groei en Krimp, 9-24.

Manacorda, M. y Moretti, E. (2006). "Why Do Most Italian Youths Live with Their Parents?: Intergenerational Transfers and Household Structure». Journal of the European Economic Association, 4(4), 800-829. <https://doi.org/10.1162/JEEA.2006.4.4.800>

Martins, N. y Villanueva, E. (2009). «Does High Cost of Mortgage Debt Explain Why Young Adults Live with Their Parents?». Journal of the European Economic Association, 7(5), 974-1010. <https://doi.org/10.1162/JEEA.2009.7.5.974>

Miret, P. (2006). «Escolarización, mercado de trabajo y emancipación familiar en España: Un análisis longitudinal a escala de Comunidad Autónoma». Papeles de Geografía, 43, 73-92.

Mitchell, B.A.; Wister, A.V. y Burch, T.K. (2017). «The Family Environment and Leaving the Parental Home». Journal of Marriage and Family, 51(3), 605-613. $<$ https://doi.org/10.2307/352160>

Modena, F. y Rondinelli, C. (s. f.). «Leaving home and housing prices: The experience of Italian youth emancipation». Banca d'Italia. Eurosistema, 818. <https://doi.org/10.1162/JEEA.2008.6.6.1109>

Moreno, A. (2012). "The transition to adulthood in Spain in a comparative perspective: The incidence of structural factors». Young, 20(1), 19-48. <https://doi.org/10.1177/110330881102000102>

Moreno, A.; López-Peláez, A. y Segado, S. (2012). La transición de los jóvenes a la vida adulta: Crisis económica y emancipación tardía. Barcelona: Obra Social "la Caixa”. Estudios Sociales, 34.

Moreno, A. y Rodríguez, E. (2012). «Informe Juventud en España 2012». Madrid: INJUVE, Instituto de la Juventud. Recuperado de <http://www.injuve.es/sites/ default/files/IJE2012_0.pdf>.

Мүктта, L. у (2012). Economic Downturns and the Failure to Launch: The Living Arrangements of Young Adults in the United States 1995-2011. Milán: USC Census Bureau.

PARISI, L. (2008). "Leaving home and the chances of being poor: The case of young people in southern European countries». Labour, 22(1), 89-114. <https://doi.org/10.1111/j.1467-9914.2008.00414.x>

Patón Casas, J.M. (2007). «Emancipación juvenil y políticas de vivienda en Europa». ACE. Architecture, City and Environment, 5, 523-554. Recuperado de <http:// upcommons.upc.edu/revistes/bitstream/2099/3709/1/DEF16_paton.pdf>.

South, S.J. y LEI, L. (2015). «Failures-to-launch and boomerang kids: Contemporary determinants of leaving and returning to the parental home». Social Forces, 94(2), 863-890.

<https://doi.org/10.1093/sf/sov064> 
Torrado, J.M. (2018). «¿Seleccionan las ciudades a su población?: Tendencias de selectividad residencial en las cabeceras metropolitanas andaluzas». Cuadernos Geográficos, 57(2), 211-236.

Whittington, L. y Peters, H. (1996). «Economic incentives for financial and residential independence». Demography, 33(1), 82-97. $<$ https://doi.org/10.2307/2061715> 